\title{
Bending "On the Rocks" - A Cocktail of Biophysical Modules to Build Endocytic Pathways
}

\author{
Ludger Johannes ${ }^{1,2}$, Christian Wunder ${ }^{1,2}$, and Patricia Bassereau ${ }^{3,4,5}$ \\ ${ }^{1}$ Institut Curie-Centre de Recherche, Traffic, Signaling and Delivery Group, 75248 Paris Cedex 05, France \\ ${ }^{2}$ CNRS UMR144, France \\ ${ }^{3}$ Institut Curie-Centre de Recherche, Membrane and Cell Functions Group, 75248 Paris Cedex 05, France \\ ${ }^{4}$ CNRS UMR168, France \\ ${ }^{5}$ Université Pierre et Marie Curie, F-75252 Paris, France \\ Correspondence: ludger.johannes@curie.fr
}

Numerous biological processes rely on endocytosis. The construction of endocytic pits is achieved by a bewildering complexity of biochemical factors that function in clathrin-dependent and -independent pathways. In this review, we argue that this complexity can be conceptualized by a deceptively small number of physical principles that fall into two broad categories: passive mechanisms, such as asymmetric transbilayer stress, scaffolding, line tension, and crowding, and active mechanisms driven by mechanochemical enzymes and/or cytoskeleton. We illustrate how the functional identity of biochemical modules depends on system parameters such as local protein density on membranes, thus explaining some of the controversy in the field. Different modules frequently operate in parallel in the same step and often are shared by apparently divergent uptake processes. The emergence of a novel endocytic classification system may thus be envisioned in which functional modules are the elementary bricks.

Endocytosis is an intracellular trafficking Eevent in which the plasma membrane is the donor compartment (Howes et al. 2010; McMahon and Boucrot 2011; Gonnord et al. 2012). Virtually any cellular function receives at some point endocytic input. Striking examples include nutrient uptake, compartmentalization and termination of intracellular signaling events, establishment of morphogen gradients, and cellular entry of pathogens and pathogenic factors. In this article, we focus our discussion on endocytic processes at the plasma membrane. It is important to point out that the principles that we will be discussing (curvature generation, motor activity, etc.) apply to other intracellular trafficking steps, notably also to endosomal sorting.

The endocytic process is also an active field of discovery concerning the molecular mechanisms of intracellular trafficking, owing to the ease at which material can be added at controlled rates and concentrations into the system, and

Editors: Sandra L. Schmid, Alexander Sorkin, and Marino Zerial

Additional Perspectives on Endocytosis available at www.cshperspectives.org

Copyright (C) 2014 Cold Spring Harbor Laboratory Press; all rights reserved; doi: 10.1101/cshperspect.a016741

Cite this article as Cold Spring Harb Perspect Biol 2014;6:a016741 
L. Johannes et al.

very early molecular events can be visualized with advanced microscopy techniques (Merrifield 2004; Kaksonen et al. 2006; Kumari et al. 2010). A confusing complexity of biochemical functions has thereby been identified, which operate in the different phases of the endocytic process: initiation, cargo recruitment, membrane bending, and scission.

Despite an ever-increasing data density, it has often proven difficult to unambiguously ascribe a functional identity to given trafficking factors or complexes, which we here term "biochemical modules," or "modules" for short. For example, BAR-domain proteins have been positioned as curvature inducers or sensors (Doherty and McMahon 2009; Frost et al. 2009). Furthermore, given molecular functions often are accomplished by several complementary modules, such as scission in the clathrin pathway-which appears to be driven by the mechanochemical enzyme dynamin (Pucadyil and Schmid 2009) — by helix-inserting epsin (Boucrot et al. 2012), and possibly also by the actin cytoskeleton (Liu et al. 2006), at least in yeast.

In this review, we argue that a true understanding of module identity requires two things. First, an exact description of the module's functional potentialities is required. This typically comes from model membrane reconstitution studies in which experimental parameters can be precisely tuned. The second requirement is the transposition of these potentialities into the cellular context in which the parameter space is determined by expression levels and activity states. As a consequence, only a limited spectrum of possible activity states will be used in the cellular context, and it is from these operationally relevant activity states that endocytic processes are built.

\section{PHYSICAL PRINCIPLES}

\section{General Physical Mechanisms to Bend Membranes}

Membrane trafficking strongly relies on the capacity of lipid membranes to bend. The energy that is required for this depends directly on the membrane's bending rigidity modulus $\kappa$ that in turn is a characteristic of membrane composition (see Box 1) (Helfrich 1973). Typical $\kappa$ values range between 10 and 60 times the thermal energy $k_{B} T$ for membranes in a fluid state (Marsh 2006). In general, acyl chain unsaturation decreases $\kappa$, whereas cholesterol and acyl chain saturation increase membrane rigidity (Rawicz et al. 2000). As an example, the bending rigidity of a membrane made from "raft lipids" (basically sphingolipids and cholesterol) and phospholipids is of the order of $60 k_{B} T$ (Roux et al. 2005), whereas it is of the order of 20$30 k_{B} T$ for a lipid mixture made of unsaturated phospholipids and cholesterol (Pan et al. 2009). Lipid bilayers have a flat geometry as long as the areas of both leaflets are equal. When the leaflets are asymmetric (i.e., the number of lipids is different or proteins are inserted in an unbalanced fashion), the membrane bends. The free energy per unit area is given by (Helfrich 1973):

$$
F_{\text {bending }}=\frac{\kappa}{2} \times\left(C-C_{0}\right)^{2} \text {, }
$$

where $C$ is the imposed membrane curvature, and $C_{0}$ is the spontaneous membrane curvature (i.e., the relaxed equilibrium curvature of the membrane in the absence of external mechanical action) (Box 2).

\section{BOX 1. BENDING MODULUS}

This parameter is a mechanical characteristic of the membrane and represents the energy required to bend it. The bending modulus depends on lipid composition. In general, membranes made from lipids with unsaturated acyl chains have a lower bending modulus than membranes made from lipids with saturated acyl chains, and cholesterol increases the bending modulus. For fluid membranes, the bending modulus ranges between 10 and $100 k T$ ( $k$ is the Boltzmann constant and $T$ is the absolute temperature, $k T=4 \times 10^{-21} \mathrm{~N} / \mathrm{m}$ at room temperature). 


\section{BOX 2. SPONTANEOUS CURVATURE}

Parameter that corresponds to the unstressed curvature of the membrane at equilibrium. Starting from a flat membrane (spontaneous curvature $C_{0}=0$ ), a nonzero spontaneous curvature can be produced by a number of mechanisms (asymmetric change in membrane area in one of the two leaflets, scaffolding, etc.; see text for details) (Figs. 1 and 2). The spontaneous curvature of a membrane depends on the surface density of curvature-generating proteins. By convention, positive spontaneous curvature refers to cases in which the membrane invaginates toward the curvature-inducing protein, and negative curvature to cases in which the membrane invaginates away from the curvature-inducing protein.

\section{Asymmetric Protein Insertion/Lipid Compaction}

In vivo, different mechanisms can produce a nonzero $C_{0}$. Proteins that insert amphipathic or hydrophobic structures from one side of the membrane into the bilayer can produce membrane bending (Fig. 1A) (Antonny 2011). The resulting effect of wedge insertion depends on protein density on the membrane, on the depth of insertion, and also on the protein's detailed shape, conical versus cylindrical (Campelo et al. 2008).

Similar effects are expected for conical transmembrane proteins (Aimon et al. 2014) or multimers of membrane proteins forming a structure with a noncylindrical shape (for instance, reticulon) (Fig. 1A) (Hu et al. 2008). At low protein density, it is expected that the

\section{A Asymmetric insertion}

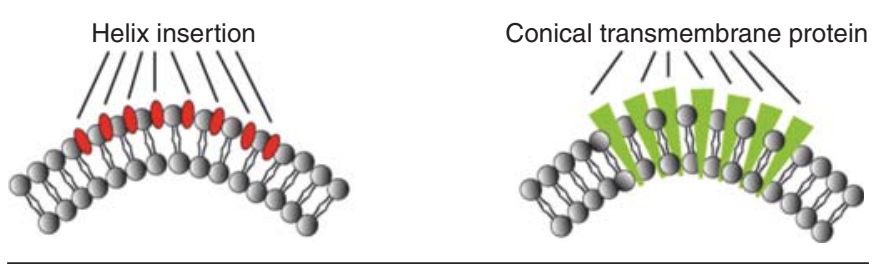

B

Lipid compaction

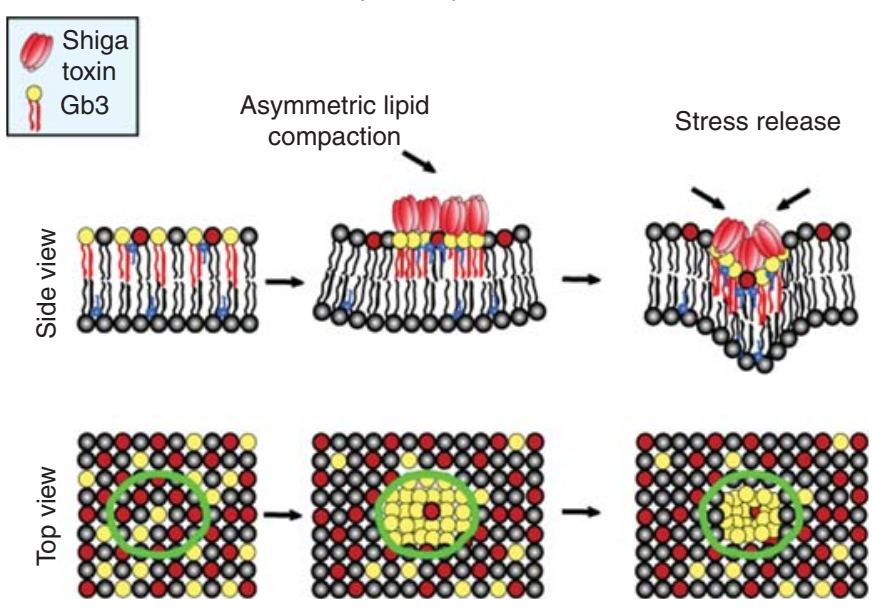

Figure 1. Membrane-bending mechanisms based on asymmetric transbilayer stress. $(A)$ Asymmetric helix insertion into one leaflet, or asymmetric shape of transmembrane proteins. (B) Lipid compaction. 
L. Johannes et al.

A

Chirality-induced budding

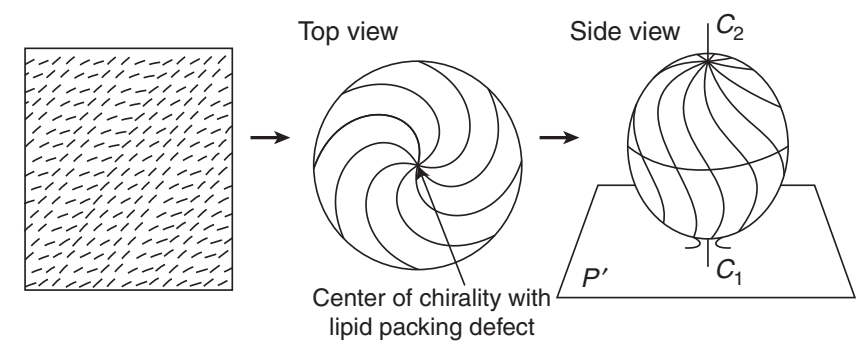

B Scaffolding
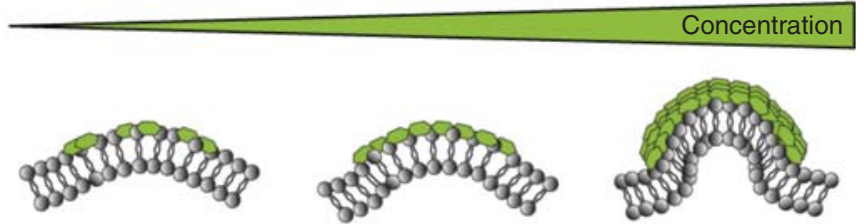

Curvature-sensing BAR domain Curvature-inducing BAR domain

C

Crowding

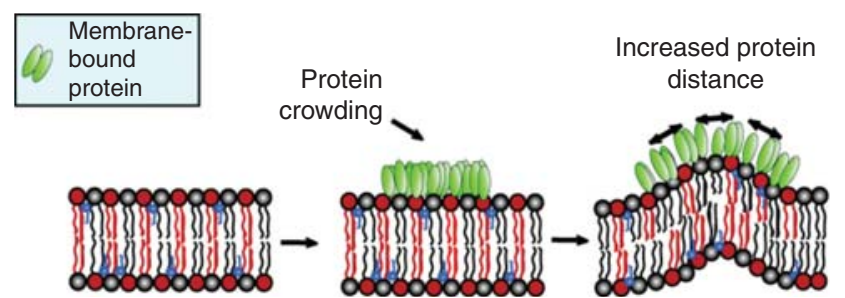

Figure 2. Membrane-bending mechanisms based on $(A)$ chirality, $(B)$ scaffolding, and $(C)$ crowding. See text for details. (Panel $A$ from Sarasij et al. 2007.)

spontaneous membrane curvature $C_{0}$ is directly proportional to the fractional density of proteins $\phi$ bound or inserted in the membrane (Leibler 1986; Campelo et al. 2008): $C_{0}(\phi)=\overline{\mathrm{C}}_{0} \phi$, with $\bar{C}_{0}$ being the effective spontaneous curvature of the protein, a molecular protein-specific parameter. In the case of transmembrane proteins, $\bar{C}_{0}$ can be related to an effective shape of the protein (Aimon et al. 2014). The clustering of such proteins would lead to the spontaneous formation of a membrane bud.

In a biological context, the directionality of membrane deformation is functionally relevant. If a cytosolic protein binds to the cytoplasmic leaflet and induces membrane bending toward the cytosolic side, this curvature is arbitrarily called "positive." In contrast, the cur- vature induced toward the cytosolic space by a protein bound to the exoplasmic leaflet is termed "negative." Although many cytosolic proteins drive positive curvature, insertions into bilayers can in principle produce any type of curvature (Campelo et al. 2008), and pathogens can exploit the induction of negative curvature at the plasma membrane to enter into, or bud out of cells (Solon et al. 2005; Römer et al. 2007; Welsch et al. 2007; Ewers et al. 2010; Rossman and Lamb 2011). In the case of toxins that specifically bind to glycosphingolipids, it has been suggested that negative curvature might be produced by the cone shape geometry of protein-lipid complexes, possibly accompanied by compaction of the lipid head groups (Fig. 1B) (Römer et al. 2007; Sens et al. 2008). Acyl chain splaying under toxin-bound lipid 
head groups would impose mechanical strain that is released by bending. The exact molecular details still need to be worked out.

Other examples for positive and negative membrane curvature-inducing proteins that function at the level of endosomes are discussed elsewhere in this collection, including Henne et al. (2013), Burd and Cullen (2014), and Gautreau et al. (2014).

\section{Chirality}

Another often-neglected effect could also account for membrane deformation: the intrinsic asymmetry (chirality) of membrane material, proteins, and/or (glyco)lipids (Fig. 2A). When such chiral molecules are organized in so-called orientation fields, topological defects emerge, leading to constraints that exist in the flat membrane state, and which can be relaxed through the formation of buds or tubules (Sarasij et al. 2007). For the example of cholera toxin it has indeed been described that its multivalent binding to the glycosphingolipid GM1 induces the emergence of a new organization of the lipid phase, termed textured phase (Watkins et al. 2011). The clustering of glycolipids or glycosylphosphatidylinositol (GPI)-anchored proteins, possibly leading to their organization in orientation fields, may be favored by their coupling to the dynamic cortical actin cytoskeleton (Goswami et al. 2008; Gowrishankar et al. 2012). The mechanisms of this coupling still need to be worked out, and may involve transmembrane proteins that themselves interact with the actin machinery via their cytosolic tails.

\section{Scaffolding}

Spontaneous membrane curvature can furthermore result from the tight binding of proteins with a rigid and curved backbone onto a membrane (Fig. 2B). Local membrane deformation and thus $\bar{C}_{0}$, results then from the adhesion between the membrane and the protein surface, as in a mold. This scaffolding process is relevant, for instance, for the BAR-domain protein superfamily (Frost et al. 2009; Mim and Unger 2012) whose backbones consist of dimers with different intrinsic curvature and orientation, depending on the specific member of the family. N-BAR proteins contain membrane-inserting amphipathic helices, in addition to BAR domains. In this case, both elements can synergistically produce curvature. Furthermore, the curvature induction capacity of a given BAR-domain protein critically depends on its local density (see section on Inducing versus Sensing).

\section{Crowding}

Recent work showed that even in the absence of bilayer insertion and scaffolding, membrane budding and tubulation can occur if membrane-bound proteins are concentrated in domains (Fig. 2C) (Stachowiak et al. 2010, 2012). Here, the driving force is a crowding mechanism in which the steric interactions between proteins or with the membrane create lateral pressure that results in spontaneous bending.

\section{Coats}

Some proteins self-assemble into rigid, curved, shell-like structures forming coats, which, bound to the membrane, impose their curvature onto the bilayer. The coat shape depends on the shape of the individual components and on the lateral interactions; it can be spherical as in the case of clathrin, COPI or COPII (McMahon and Mills 2004; Stagg et al. 2007), or tubular-like for dynamin (Schmid and Frolov 2011; Ferguson and De Camilli 2012), and F-BAR-domain proteins (e.g., FBP17 or FCHo in human cells) (Masuda and Mochizuki 2010). The typical radii for these structures are of the order of 50 $120 \mathrm{~nm}$ for the spherical coats. For dynamin, the membrane tube radius is $10 \mathrm{~nm}$, whereas the radii imposed by F-BAR domains are larger ranging from typically $30 \mathrm{~nm}$ to $>50 \mathrm{~nm}$ (Henne et al. 2007; Frost et al. 2008). Of note, the use of the coat term in the context of dynamin and F-BAR-domain-containing proteins may appear unconventional. Yet, these proteins clearly have the capacity to self-assemble onto membranes to form a rigid structure, and therefore conform to the definition of coats. 
L. Johannes et al.

\section{Local Pulling Force}

Ultimately, membrane tubes can be extended when applying a local force, the amplitude of which (in the order of up to tens of piconewtons) depends on the membrane's bending rigidity and its lateral tension (Derényi et al. 2002). Molecular motors bound to the membrane (such as kinesins and myosins) and moving along cytoskeleton filaments (microtubules in the case of kinesins, and actin in the case of myosins) have been shown to be able to produce such forces (Fig. 3) (Roux et al. 2002; Koster et al. 2003). The tubule radii are quite narrow, usually well below $200 \mathrm{~nm}$, and sometimes as little as $10 \mathrm{~nm}$.

\section{Line Tension}

We have listed here a series of bending mechanisms involving proteins inserted into or bound onto membranes. Early theoretical predictions (see, for instance, Julicher and Lipowsky 1993) and later in vitro experiments using giant liposomes (e.g., Baumgart et al. 2003) have shown that membranes made of pure lipid mixtures can also bend locally, in the absence of proteins and transbilayer asymmetry, providing that lipid domains are present (Box 3). The force that produces membrane bending in this case is the line tension $\gamma$ at domain interfaces, which results from misalignments (e.g., curvature or height mismatches) that cause hydrophobic acyl chain segments to be exposed to bulk water molecules or hydrophilic head groups from neighboring lipids (Fig. 4A) (Semrau and Schmidt 2009; Johannes and Mayor 2010). An example is long acyl chain glycosphingolipids (GSLs) that are clustered by bacterial toxins to form a domain that is thicker than the bulk membrane (Römer et al. 2007). Depending on lipid composition and temperature, line tension can vary between 0.1 and $10 \mathrm{pN}$ in in vitro systems (Garcia-Saez et al. 2007; Tian et al. 2007). Line tension produces a constriction in the attempt to reduce the perimeter of the domain boundary and, thereby, the energy penalty. This effect is counterbalanced by the bending energy of the lipid domain and membrane tension that tends to flatten the membrane. If membrane tension is strongly reduced by deflating the giant liposome, line tension can be sufficient to allow the membrane domain to bud and detach from the limiting membrane (Box 4) (Lipowsky 1993; P Bassereau, unpubl.).

We can estimate the smallest size $R_{\min }$ of the bud that can be formed by this process. The energy required to generate a vesicle can be calculated by integrating Equation 1 on a sphere, which yields $F_{\text {bending }}=8 \pi \kappa$, independently of the size of the bud. The line-tension energy is $F_{\text {line }}=2 \pi R_{\min } \gamma$. The lowest size corresponds to the bud formed at zero tension, which can be estimated as,

$$
R_{\min } \approx \frac{4 \kappa}{\gamma} \approx 300 \mathrm{~nm}
$$

for typical membrane parameters of $\kappa \approx 40 k_{B} T$ and $\gamma \approx 2 \mathrm{pN}$ (Sens et al. 2008). Larger buds are expected to form as soon as membrane tension increases. This very simplistic estimate implies that line tension alone cannot explain the formation of narrow curvature radii that characterize transport carriers on cells, and that proteins are necessary for biological membrane bending.

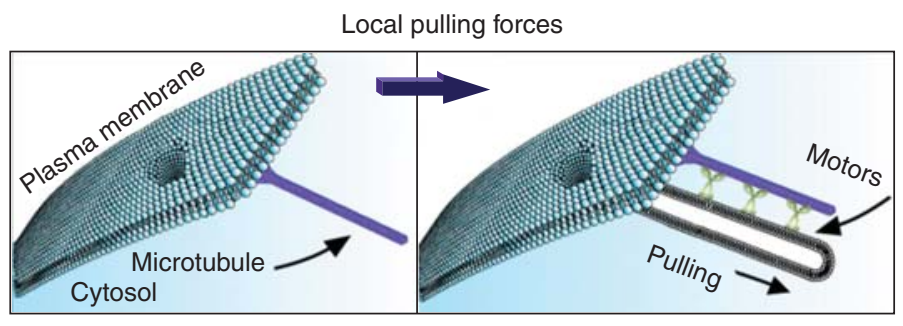

Figure 3. Motor-driven formation of membrane tubules on microtubule tracks. 


\section{BOX 3. LINE TENSION}

Line tension is related to the energy cost at domain interfaces. For instance, if a domain is thicker than the surrounding bulk membrane, lipids are misaligned at the domain interface, which leads to unfavorable contacts between hydrophobic acyl chains and hydrophilic head groups from neighboring lipids, or water molecules from the solvent (Fig. 4A). Line tension in lipid membranes has been measured with values ranging between 0.1 and $10 \mathrm{pN}$.

\section{Control of the Carrier Size}

Most proteins that deform membranes through the generation of spontaneous membrane curvature or by a crowding mechanism produce elongated membrane tubules and not spherical buds. Tubule diameter is in principle related to the spontaneous curvature of the protein, but no constraints besides membrane tension and total protein quantity intrinsically regulate the length of these tubules. This is also true for coat-forming proteins polymerizing as cylindrical structures (i.e., BAR-domain proteins). Yet, there are many cases in which the number of cargoes per transport carrier has to be strictly controlled, such as for synaptic vesicle formation, or the internalization of receptors or adhesion molecules. This requirement could
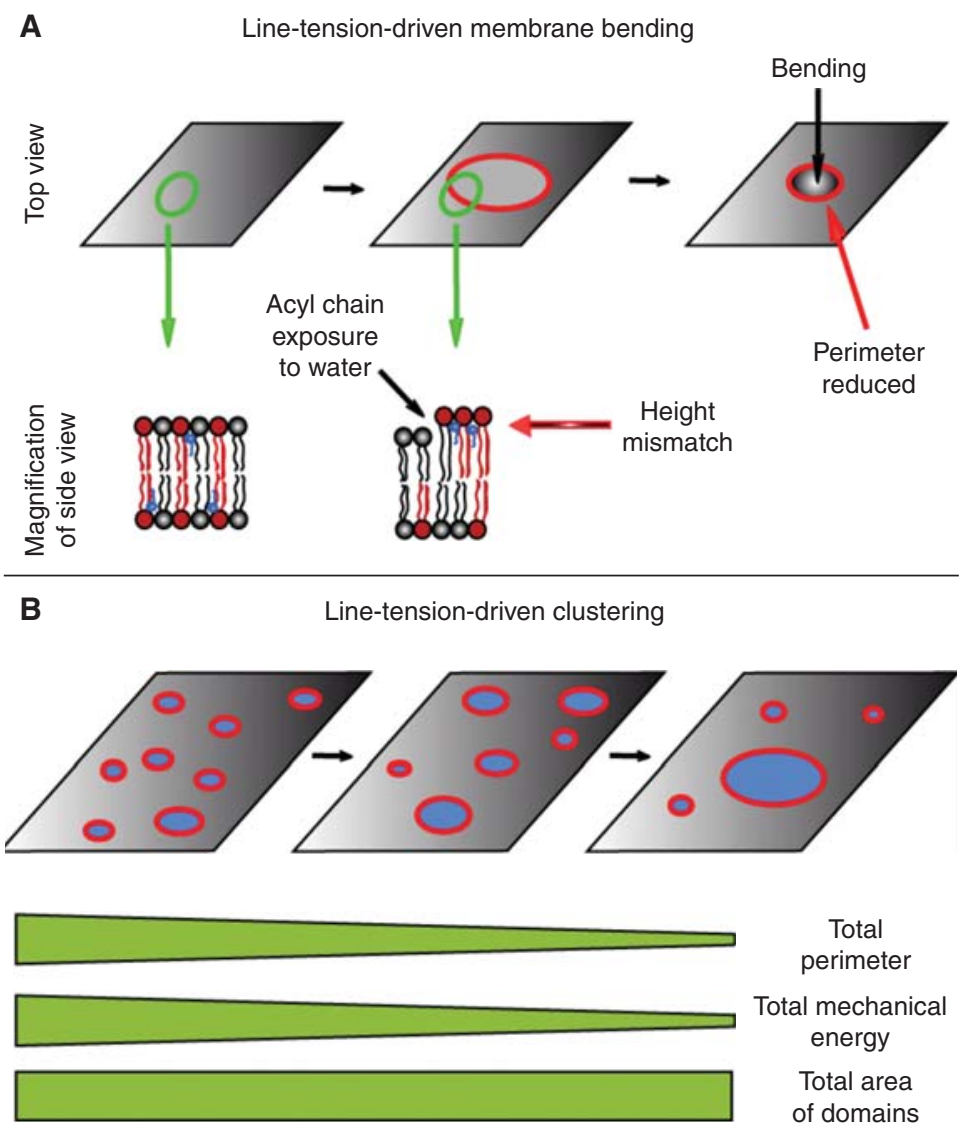

Figure 4. Line tension. (A) Membrane bending. (B) Membrane-mediated clustering. 
L. Johannes et al.

\section{BOX 4. MEMBRANE-MEDIATED CLUSTERING MECHANISM}

Proteins can cluster in the absence of direct protein-protein interactions. Such membrane-mediated clustering can be driven by hydrophobic mismatch in the case of transmembrane proteins (i.e., the thickness of the lipid membrane does not match the height of the hydrophobic part of the transmembrane protein), and more generally by a reduction of the line tension (Fig. 4B). Membrane fluctuations can also drive clustering owing to entropic effects.

in principle be met in two ways: a tight timing of scission or additional mechanical constraints by which spontaneous curvature sets the size of the spherical carrier (its diameter). It is the latter solution that appears to have been evolved preferentially with the emergence of rigid coats that provide high fidelity for transport carrier formation at the plasma membrane (e.g., synaptic vesicles for quantal neurotransmitter release), but also for trafficking between the endoplasmic reticulum (ER) and Golgi. The ancestral tubular carrier structures remain in use at many intracellular trafficking interfaces for less tightly normalized functions.

\section{Scission}

A lipid membrane can be disrupted upon application of a high stretching force. Lysis tension typically ranges between $10^{-3}$ and $10^{-2} \mathrm{~N} / \mathrm{m}$ (Evans and Smith 2011). In biological systems, detachment of the transport carrier from the limiting membrane occurs in a nonleaky fashion, which is achieved, possibly via a hemifission state, by mechanisms leading to the squeezing or pinching of the neck of the spherical bud or, locally, of a tubule.

\section{Line Tension}

The scission of a bud or tubule requires overcoming an energy barrier in the process. In a pure lipid mixture, line tension at the edges of lipid domains can provide a constriction force (Fig. 5), as discussed above. It has indeed been argued on theoretical and experimental grounds that membrane tubules can undergo line-tension-driven scission along the domain edge (Allain et al. 2004; Roux et al. 2005).

\section{Membrane Tension and Pulling Forces}

Interestingly, the increase of membrane tension that results from a reduced tubule radius considerably decreases the time for scission after phase separation (Allain et al. 2004). In vivo, pulling forces that are applied to the budding structure by molecular motors or polymerizing actin filaments are expected to reduce the energy barrier involved in neck or tubule fission (Liu et al. 2006).

\section{Scission Proteins}

Dynamin or dynamin-like proteins induce membrane scission by converting chemical energy of GTP into a squeezing force on the membrane neck (Chappie et al. 2011; Campelo and Malhotra 2012; Shnyrova et al. 2013). A recent paper has shed light on the fission mechanism (Morlot et al. 2012). Twisting of the helical dynamin structure results in local constriction of

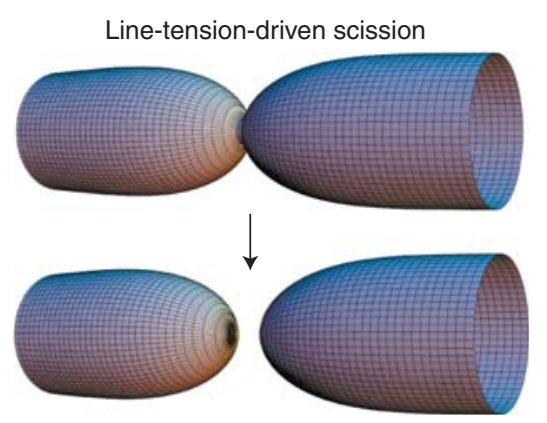

Figure 5. Line-tension-driven scission. The formation of a lipid domain in a membrane tubule leads to spontaneous constriction at domain interfaces to reduce line tension. Schematically, a light blue domain (left) is juxtaposed to a dark blue domain (right). (Courtesy of Storm et al. 2004.) 
the membrane under the dynamin coat, which increases the local membrane elastic energy at the edge of the dynamin polymer and significantly reduces the energy barrier for fission. This situation is in principle similar to line tension at domain interfaces that we have discussed above. The boundary forces here result from the discontinuity between the constricted tube coated with dynamin, and the noncoated tube with a larger radius. Scission then takes place at this location with a kinetics essentially controlled by the membrane elasticity (bending rigidity and membrane tension). Another set of experiments proposes that membrane constriction by dynamin rings is assisted by the tilt of the $\mathrm{PH}$ domain upon GTP hydrolysis, creating a wedge effect (Shnyrova et al. 2013). These mechanisms may work alone or in combination, and may apply to other proteins or protein assemblies that use nucleotide hydrolysis to constrict the membrane.

\section{Helix Insertion}

Local destabilization of the membrane upon hydrophobic insertion is an alternative mechanism for fission. In a recent paper, a combination of thermodynamic, in vitro and in vivo, approaches was used to show that increasing the density of insertions in the membrane drives membrane deformation from tubulation to fission, independently of nucleotide hydrolysis (Boucrot et al. 2012). The fact that in N-BAR proteins the amphipathic helices are attached to BAR domains limits the density of insertions into the membrane and thereby the fission efficiency.

\section{Experimental Systems}

As seen above, different mechanisms account for membrane bending and scission. To discriminate stringently between these, in vitro experiments with purified proteins and model membranes are used. A typical approach consists in incubating small 50- to $100-\mathrm{nm}$-diameter liposomes in the presence of proteins whose potential to bend membranes is under scrutiny. Membrane shape changes are monitored by electron microscopy (see, for instance, Peter et al. 2004;
Mattila et al. 2007). In the case of organized coats, structural data can be deduced (Danino et al. 2004; Frost et al. 2008). In the presence of high-protein concentrations, the spontaneous curvature of the ligand can in principle be deduced from the diameter of the tubules (Takei et al. 1998).

In some cases, the effect of protein binding on membrane curvature can be addressed with small liposomes and light scattering (Antonny 2011) or quantitative high throughput fluorescence microscopy (Bhatia et al. 2010). However, under these conditions, one only has access to bulk protein concentrations, which makes it difficult to compare the data with theoretical models. Giant liposomes (also called GUVs, for "giant unilamellar vesicles") represent an experimental system that has a number of advantages over the bulk approaches. GUVs enable the monitoring of membrane deformation and protein distribution with optical microscopy, and provide at the same time control over membrane tension (Sens et al. 2008). Membrane nanotubes can be pulled with controlled curvature radii, and the mechanical action of proteins on membranes can be deduced from force measurements and compared with theoretical models (Sorre et al. 2012). Protein distribution can be tuned as a function of membrane curvature by continuous changing of the tube radius under concomitant observation by fluorescence microscopy (Sorre et al. 2009, 2012; Roux et al. 2010; Singh et al. 2012). Membrane fission can be followed in real time and analyzed in detail (Römer et al. 2010; Morlot et al. 2012). Membrane tubes can also be pulled from a planar lipid bilayer using a patch-clamp micropipette (Bashkirov et al. 2008), allowing for electrophysiology measurements. The constrictive effect of proteins can then be monitored via the conductivity of the nanotube.

Tension-free tubules can be obtained after protein binding either onto lipid-coated planar glass substrates, or lipid-coated beads (Roux et al. 2006; Pucadyil and Schmid 2008). In these setups, many tubes can be observed at the same time.

In this section of the review, we have seen how various protein modules drive membrane 
L. Johannes et al.

curvature changes, exploiting a limited set of physical principles. In the following section, we discuss some cell biological aspects of endocytosis from the perspective of these physical considerations.

\section{CELL BIOLOGY OF ENDOCYTIC MEMBRANE BENDING}

From Apparently Simple to Complex: Construction of Endocytic Pits

In going from simple to more evolved organisms, an apparent complexification of the endocytic machinery can be observed. In the clathrin pathway of mammalian cells, several molecules have the potential to participate in membrane bending, including clathrin itself via a rigid coat effect (Dannhauser and Ungewickell 2012), BAR-domain-containing scaffolding proteins (Henne et al. 2010; Cocucci et al. 2012), and helix-inserting proteins such as epsins (Ford et al. 2002). In comparison, the endocytic pathway in yeast appears to be simpler. Clathrin is not strictly required, and BAR-domain proteins come in at late stages of endocytic membrane invagination for a likely function in scission that is mostly driven by actin (Kaksonen et al. 2005). For certain bacterial toxins and animal viruses it has even been argued that initial endocytic membrane bending does not strictly require cytosolic machinery and is induced by GSL reorganization (Römer et al. 2007; Ewers et al. 2010). In this case, the signal that is sent to the cytosol appears to be high membrane curvature, which would then be recognized by cytosolic machinery such as actin (Römer et al. 2010) and BAR-domain proteins for further processing into the cell. Evolutionary complexification thereby appears to be achieved by an increasing number of functional modules that contribute to the bending process.

Does multimodality represent redundancy? At this stage, no clear answer can be given. The depletion of all three epsins from mammalian cells prevents clathrin-dependent transferrin endocytosis (Boucrot et al. 2012). This block was not at the level of membrane bending, but rather for scission. Although this has allowed identifying an unexpected function of epsins is in the latter reaction (see below), it is still likely that epsins also contribute to initial bending, but in a redundant manner. Such redundancy might also explain why BAR-domain proteins such as FCHos (Henne et al. 2010; Cocucci et al. 2012) have variable phenotypes in different experimental systems, and why genetic deletion of well-studied BAR-domain proteins such as amphiphysin does not produce a generalized uptake phenotype in the clathrin pathway (Di Paolo et al. 2002). Redundancy likely improves the robustness of the endocytic system, which gains importance as organisms become multicellular and need to integrate the capacity of sending and receiving precisely defined signals.

The increased complexity of endocytic membrane-bending mechanisms results in marked changes concerning the morphology of endocytic carriers. Uptake structures that are generated by clathrin-independent endocytosis are notoriously pleomorphic (Hansen and Nichols 2009), and often have tubular or cisternal shapes (Kirkham et al. 2005; Massol et al. 2005; Römer et al. 2007). In contrast, clathrin-coated vesicles are of defined sizes between $50 \mathrm{~nm}$ (synaptic vesicles) and 80-120 nm (Fotin et al. 2004). This normalization in size is achieved at the price of bringing high amounts of clathrin, adaptors, and accessory proteins onto the membrane whose intrinsic self-organization capacity impinges the preferred spherical shape of the vesicle. In all cases of clathrin- and caveolinindependent endocytosis, highly organized coatlike structures could not be detected at sites of membrane invagination (Howes et al. 2010), which likely explains the pleomorphic nature of resulting transport carriers. As we have seen above, the bacterial Shiga toxin (Römer et al. 2007), cholera toxin, and the polyoma virus SV40 (Ewers et al. 2010) reorganize GSLs in the exoplasmic leaflet of the plasma membrane, and thereby drive the clathrin-independent formation of narrow tubular endocytic invaginations. It is not yet clear whether similar mechanisms also operate in the case of clathrin-independent uptake of endogenous proteins.

The simple-to-complex scheme becomes more convoluted when looking at actin. Recent 
time-resolved electron tomography studies show that, in yeast, actin forces appear to be required for the formation of initial curvature (Kukulski et al. 2012, but see also Idrissi et al. 2012), and to push the membrane tubule toward the interior. The specificity of the yeast system is that the membrane invagination process has to overcome high membrane tension owing to turgor pressure. Correspondingly, actin in the mammalian clathrin pathway appears to be strictly required only in specific conditions of high membrane tension caused experimentally by osmotic swelling or mechanical stretching (Boulant et al. 2011). In yeast, an additional element emerges from the fact that the organism is surrounded by a rigid cell wall, like in plants. We can thus hypothesize that the actin cytoskeleton can exert inward force by pushing onto this cell wall, in contrast to mammalian cells in which the counteracting force would be minimal owing to the flexibility of the plasma membrane.

In clathrin-independent endocytosis in higher eukaryotes, actin is clearly a key element (Howes et al. 2010), likely by favoring receptor clustering (Goswami et al. 2008) and scission (Römer et al. 2010). Whether actin also drives membrane bending directly remains to be analyzed.

Inducing versus Sensing

An important question that has caused some confusion is whether BAR-domain proteins are curvature sensors or inducers. Already in the pioneering paper on the structure of the BAR domain of amphiphysin, both aspects were brought up (Peter et al. 2004). Subsequent studies clearly documented the appearance of tubular structures upon overexpression of BAR-domain proteins (reviewed in Frost et al. 2009). A recent study has allowed conceptualizing this apparent contradiction (Sorre et al. 2012). Using amphiphysin as a model, it was shown that the protein's mechanical effects on a membrane tubule depend on protein density (Fig. 2B). At very low densities, curvature sensing prevails (i.e., strong enrichment of the protein on highly curved membrane segments), and no mechanical effects are observed. In con- trast, a scaffold is formed around the tubule at elevated amphiphysin densities, imposing very high mechanical strain onto the membrane. These findings illustrate that one needs to integrate information on local protein densities on membranes when reasoning about the question of curvature sensing or induction in the cellular context.

From Apparently Simple to Complex: Membrane Scission

Biological membrane scission is another example for which the from-simple-to-complex paradigm appears to apply. The GTPase dynamin has received the most attention in this context, owing to its preponderant role in the clathrin pathway. Dynamin clearly is a mechanoenzyme (Doherty and McMahon 2009; Mettlen et al. 2009; Chappie et al. 2011). Recent studies have shown that scission occurs spontaneously at the interface of the dynamin lattice and the bare membrane once dynamin has reduced the energy barrier sufficiently by constricting the membrane tubule or bud neck (Morlot et al. 2012).

Yet dynamin is not the whole scission story, even in the clathrin pathway. Under certain experimental conditions of overexpression, epsins via their helix insertion capacity can functionally replace dynamin in the clathrin-dependent uptake of transferrin (Boucrot et al. 2012). In this study, an intricate interplay has been revealed in which BAR domains limit the scission process that is driven by membrane-inserting helices. It remains yet to be determined how this general finding applies to other endocytic processes.

Many of the clathrin-independent endocytosis processes are dynamin independent, or at least not strictly reliant on dynamin. The prime example is yeast. Here, BAR-domain proteins are recruited at late stages before scission, and it has been argued on theoretical grounds that a scaffold of BAR domains would create a lipid domain whose interface forces then drive the spontaneous squeezing of the invaginated tubule leading to scission (Fig. 5) (Liu et al. 2009). As we have seen above, physicists call such domain boundary forces line tension, 
L. Johannes et al.

and theoretical and experimental studies had already linked line tension to scission (Allain et al. 2004; Roux et al. 2005; Liu et al. 2006). The most direct evidence for a function of line tension in biological membrane scission comes from the Shiga toxin system. In a study on model and cell membranes it was shown that Shiga toxin-containing endocytic invaginations are poised such that an appropriate trigger induces membrane reorganization leading to line tension and scission (Römer et al. 2010). In cells, this trigger appears to be actin, whose binding to lipids can indeed change the phase behavior of a membrane system (Liu and Fletcher 2006). Whether this type of scission mechanism also contributes to the clathrin pathway is a possibility that still needs to be addressed directly.

\section{A Rafty Bending Business}

It is now clearly established that the plasma membrane is compartmentalized. Such compartmentalization appears to be driven by active processes linked to the actin cytoskeleton (Gowrishankar et al. 2012). Active membrane domains could in principle lead to the spontaneous formation of membrane buds, owing to the tendency of the system to reduce the line-tension energy penalty that exists at domain interfaces (Fig. 4A) (Baumgart et al. 2003; Bacia et al. 2005). However, the resulting curvature radii are in the micrometer range, and cannot by themselves explain the formation of narrow invaginations that characterize endocytic pits.

Membrane domains that are generated by active mechanisms could favor the formation of narrow tubules if the domain material itself contributed to bending. There are several ways how that could occur, including the clustering of proteins or lipids whose intrinsic shapes (reviewed in McMahon and Gallop 2005) or orientations (Sarasij et al. 2007) tune membrane curvature (Figs. 1 and 2). However, one needs to consider whether sufficiently stable molecular assemblies can be achieved by purely actin-driven clustering. The example of GSLs may be chosen to illustrate this point. The reorganization of cellular GSLs by bacterial Shiga (Römer et al. 2007) and cholera (Ewers et al. 2010) toxins leads to membrane bending and the formation of narrow tubular endocytic membrane invaginations. In principle, one might envision that actin dynamics on the cytosolic leaflet of the membrane might cause a similar (glyco)sphingolipid reorganization to drive membrane bending in raft lipid-dependent pathways like the CLIC/GEEC formation process (reviewed in Chadda et al. 2007). In such a case, one must assume the existence of tight transbilayer coupling mechanisms that yet need to be described in further detail. Another possibility is the spatiotemporal coupling between active actin-driven and passive membrane-mediated clustering mechanisms (Box 4). In this scenario, an interplay between active contractility, remodeling of the cytoskeleton, and transbilayer coupling results in transient focusing of passive molecules (Chaudhuri et al. 2011), such as GSLtoxin complexes. These GSL-toxin complexes could then cluster further by membrane-mediated mechanisms favored by line-tension forces resulting from packaging defaults such as height and curvature mismatches (Fig. 4A) (Johannes and Mayor 2010). The efficacy of toxin-driven membrane bending would thereby be increased, notably at low bulk toxin concentrations, as one would expect to find in the pathological situation. Whether this active-passive coupling mechanism actually applies to the toxins, and, if so, whether it has some general bearing on clathrin-independent endocytosis, remains to be addressed directly.

The concerted interplay between active and passive clustering mechanisms of raft-type materials might also favor the creation of membrane environments that are poised to carry out specific biological functions. For example, the sorting of lipids has been found, on theoretical and experimental grounds, to be most efficient in membrane systems that are close to phase separation (Sorre et al. 2009; Tian and Baumgart 2009). This is explained by lipid-lipid interactions that become dominant over mixing entropy as membranes approach lipiddemixing conditions. Of interest, GSLs can be cosorted under such conditions (Safouane et al. 2010), which may contribute to the generation of specific compositional environments that are 
programmed to carry out downstream functions. A striking example is the above-mentioned actin-driven scission process on Shiga toxin-induced membrane invaginations (Römer et al. 2010). Here, tubular endocytic invaginations at physiological temperature are very close to demixing. An appropriate trigger leads to domain formation, line-tension-driven tubule squeezing, and spontaneous scission. In other words, by inducing tubular plasma membrane invaginations with yet-to-be defined specific compositions, Shiga toxin preprograms the next step of its entry into cells, which is tubule scission. At first sight, it might appear surprising to propose that biological membrane compositions with thousands of proteins and lipids could be tuned to position the system in such states close to demixing. Exactly such events have recently been described however (Heimburg and Jackson 2005; Lingwood et al. 2008; Polozov et al. 2008). Compositional tuning opens unprecedented opportunities to biochemically impose, in many physiological contexts, the concept of induced domain formation as a driver for cell biological functions.

\section{STAGING}

Clathrin-mediated endocytosis appears to involve many of the functional modules that we have described so far to achieve budding in a very robust manner. The time sequence of this process is highly orchestrated (Taylor et al. 2011; see also Merrifield and Kaksonen 2014). No physical model exists so far to globally describe how these modules could be functionally coupled. A simple biochemical view would be that the binding sequence is driven by the sequential recruitment of protein modules. However, we argue that the coupling between curvature and protein density on the membranes can produce the time sequence for the recruitment of downstream module partners. The shaping of the membrane induced by the first deforming modules could be the signal that triggers recruitment of the next modules. Such a model requires that the modules involved in fission should appear last for correct budding, thus involving proteins either with a low density on the membrane (controlled by a reduced affinity for the membrane, or a low bulk concentration) or alternatively that are poor curvature sensors.

In vitro experiments on amphiphysin and dynamin have pointed toward curvature as a very efficient signal at moderate protein densities (Roux et al. 2010; Sorre et al. 2012). Moreover, feedback between different modules can exist as it has been described for dynamin, actin, and N-BAR proteins that can cooperatively work to efficiently catalyze membrane scission (Taylor et al. 2012). Such feedback is even more difficult to include in a physical modeling. Nevertheless, it is urgent to better understand how/why the first shaping module is recruited to bend membrane. Recent live-cell total internal reflection fluorescence microscopy (TIRF) imaging experiments with single-molecule sensitivity on clathrin-coated pits of mammalian cells have revealed that recruitment of two AP2 adaptors to the PIP2-containing membrane, stabilized by one clathrin triskelion with oligomerization capacity, can provide the first brick for further coat recruitment and subsequent bending (Cocucci et al. 2012). In yeast, a different sequence occurs as shown by experiments correlating $3 \mathrm{D}$ electron microscopy and fluorescence microscopy (Kukulski et al. 2011, 2012). Clathrin binding does not induce bending, which instead is produced by the actin network. However, immunogold labeling with high-resolution transmission electron microscopy (EM) have challenged this view and suggested that the branched actin network is involved in the elongation of an existing invagination and not in the initial bending (Idrissi et al. 2012). This difference may have arisen from different sensitivities of the methods that were used in both studies to detect initial membrane deformation.

\section{CONCLUDING REMARKS AND FUTURE PERSPECTIVES}

Classification of endocytic pathways has been a challenge ever since the discovery of clathrinindependent uptake processes. In this review, we have argued that a limited set of physical principles can be used to group biochemical machinery into functional modules. We anticipate 
L. Johannes et al.

that individual modules will be shared between different endocytic events, and that specificity within a given uptake process arises from defined cocktails of modules. Based on a true understanding of how these modules function, it should become possible to assign them to specific uptake processes, and more precisely to specific steps within the uptake process (initiation, bending, invagination ...). Such classification of endocytic events would thus consist of a chain of defined module identity, rather than a reductionist limitation to one functional element.

The key aspect here is on mechanistic understanding in terms of physical/mechanical principles. We believe that this must come from a combination of approaches that range from model membrane reconstitution with precise control over experimental conditions and access to parameters such as forces that are difficult to measure in complex systems, to systems biology explorations of integrated circuits. The strive for mechanistic understanding calls for interdisciplinary expertise from physics for concepts and workstations for quantitative measurements, from chemistry for tailor-made tools, and from biology for relevant working hypotheses and their exploration into the physiological and pathological context of cells and organisms.

The back-assignment of mechanistic conclusions from in vitro explorations to the cell and organism situation is of critical importance. In vitro experiments should be strictly quantitative, consistent between experimental conditions, and referring to the in vivo concentrations. To illustrate this point, one may recall that BARdomain proteins like amphiphysin sense or induce curvature, depending on their concentration on membranes (Sorre et al. 2012). For the assignment of their module identity within a given endocytic program, it is thus of critical importance to have access to concentration values within the context of the cell. The advent of quantitative imaging approaches holds out the promise of accessing this type of information in both a dynamic and highly localized manner.

The assignment of module identity within endocytic pathways is rendered complex by additional considerations. A functional interplay between modules is one of these. As we have seen, actin (via line tension) (Stechmann et al. 2010) and dynamin (as a mechanoenzyme) (Chappie et al. 2011) contribute to scission in their own right. They possibly also interact functionally if it is confirmed in vivo that dynamin activity is enhanced on tensed membranes (Roux et al. 2006; Ferguson et al. 2009). Another source for complexity comes from the mechanical state of the membrane substrate itself. For example, membrane tension is buffered by caveolae (Sinha et al. 2011), and influences endocytic processes (Gauthier et al. 2012). As cell biologists dig their way deeper into these complexities, they increasingly meet and team up with physicists and chemists who have become interested in biological materials as fertile substrates for their own scientific imagination.

\section{ACKNOWLEDGMENTS}

The work in the authors' laboratories on subjects related to the current review is supported by Agence Nationale pour la Recherche (ANR09-BLAN-283, ANR-11 BSV2 018 03, ANR-11 BSV2 014 03) and Institut National du Cancer (PLBIO11-022-IDF-JOHANNES). The Bassereau and Johannes teams are members of Labex CelTisPhyBio. A Marie Curie International Reintegration Grant within the Seventh European Union Framework Programme (FP7-RG277078) supports C.W.

\section{REFERENCES}

* Reference is also in this collection.

Aimon S, Callan-Jones A, Berthaud A, Pinot M, Toombes GE, Bassereau P. 2014. Membrane shape modulates transmembrane protein distribution. Dev Cell (to be published).

Allain JM, Storm C, Roux A, Ben Amar M, Joanny JF. 2004. Fission of a multiphase membrane tube. Phys Rev Lett 93: 158104.

Antonny B. 2011. Mechanisms of membrane curvature sensing. Annu Rev Biochem 80: 101-123.

Bacia K, Schwille P, Kurzchalia TV. 2005. Sterol structure determines the separation of phases and the curvature of the liquid-ordered phase in model membranes. Proc Natl Acad Sci 102: 3272-3277.

Bashkirov PV, Akimov SA, Evseev AI, Schmid SL, Zimmerberg J, Frolov VA. 2008. GTPase cycle of dynamin is coupled to membrane squeeze and release, leading to spontaneous fission. Cell 135: 1276-1286. 
Baumgart T, Hess ST, Webb WW. 2003. Imaging coexisting fluid domains in biomembrane models coupling curvature and line tension. Nature 425: 821-824.

Bhatia VK, Hatzakis NS, Stamou D. 2010. A unifying mechanism accounts for sensing of membrane curvature by BAR domains, amphipathic helices and membrane-anchored proteins. Sem Cell Dev Biol 21: 381390.

Boucrot E, Pick A, Camdere G, Liska N, Evergren E, McMahon HT, Kozlov MM. 2012. Membrane fission is promoted by insertion of amphipathic helices and is restricted by crescent BAR domains. Cell 149: 124-136.

Boulant S, Kural C, Zeeh JC, Ubelmann F, Kirchhausen T. 2011. Actin dynamics counteract membrane tension during clathrin-mediated endocytosis. Nat Cell Biol 13: 1124-1131.

* Burd C, Cullen PJ. 2014. Retromer: A master conductor of endosome sorting. Cold Spring Harb Perspect Biol doi: 10.1101/cshperspect.a016774.

Campelo F, Malhotra V. 2012. Membrane fission: The biogenesis of transport carriers. Ann Rev Biochem 81: 407427.

Campelo F, McMahon HT, Kozlov MM. 2008. The hydrophobic insertion mechanism of membrane curvature generation by proteins. Biophys J 95: 2325-2339.

Chadda R, Howes MT, Plowman SJ, Hancock JF, Parton RG, Mayor S. 2007. Cholesterol-sensitive Cdc42 activation regulates actin polymerization for endocytosis via the GEEC pathway. Traffic 8: 702-717.

Chappie JS, Mears JA, Fang S, Leonard M, Schmid SL, Milligan RA, Hinshaw JE, Dyda F. 2011. A pseudoatomic model of the dynamin polymer identifies a hydrolysisdependent powerstroke. Cell 147: 209-222.

Chaudhuri A, Bhattacharya B, Gowrishankar K, Mayor S, Rao M. 2011. Spatiotemporal regulation of chemical reactions by active cytoskeletal remodeling. Proc Natl Acad Sci 108: 14825-14830.

Cocucci E, Aguet F, Boulant S, Kirchhausen T. 2012. The first five seconds in the life of a clathrin-coated pit. Cell 150: 495-507.

Danino D, Moon KH, Hinshaw JE. 2004. Rapid constriction of lipid bilayers by the mechanochemical enzyme dynamin. J Struct Biol 147: 259-267.

Dannhauser PN, Ungewickell EJ. 2012. Reconstitution of clathrin-coated bud and vesicle formation with minimal components. Nat Cell Biol 14: 634-639.

Derényi I, Jülicher F, Prost J. 2002. Formation and interaction of membrane tubes. Phys Rev Lett 88: 238101.

Di Paolo G, Sankaranarayanan S, Wenk MR, Daniell L, Perucco E, Caldarone BJ, Flavell R, Picciotto MR, Ryan TA, Cremona O, et al. 2002. Decreased synaptic vesicle recycling efficiency and cognitive deficits in amphiphysin 1 knockout mice. Neuron 33: 789-804.

Doherty GJ, McMahon HT. 2009. Mechanisms of endocytosis. Annu Rev Biochem 78: 857-902.

Evans E, Smith BA. 2011. Kinetics of hole nucleation in biomembrane rupture. New J Phys 13: 095010.

Ewers H, Römer W, Smith AE, Bacia K, Dmitrieff S, Chai W, Mancini R, Kartenbeck J, Chambon V, Berland L, et al. 2010. GM1 structure determines SV40-induced mem- brane invagination and infection. Nat Cell Biol 12: 1118

Ferguson SM, De Camilli P. 2012. Dynamin, a membraneremodelling GTPase. Nat Rev Mol Cell Biol 13: 75-88.

Ferguson S, Raimondi A, Paradise S, Shen H, Mesaki K, Ferguson A, Destaing O, Ko G, Takasaki J, Cremona O, et al. 2009. Coordinated actions of actin and BAR proteins upstream of dynamin at endocytic clathrin-coated pits. Dev Cell 17: 811-822.

Ford MG, Mills IG, Peter BJ, Vallis Y, Praefcke GJ, Evans PR, McMahon HT. 2002. Curvature of clathrin-coated pits driven by epsin. Nature 419: 361-366.

Fotin A, Cheng Y, Sliz P, Grigorieff N, Harrison SC, Kirchhausen T, Walz T. 2004. Molecular model for a complete clathrin lattice from electron cryomicroscopy. Nature 432: 573-579.

Frost A, Perera R, Roux A, Spasov K, Destaing O, Egelman EH, De Camilli P, Unger VM. 2008. Structural basis of membrane invagination by F-BAR domains. Cell 132: 807-817.

Frost A, Unger VM, De Camilli P. 2009. The BAR domain superfamily: Membrane-molding macromolecules. Cell 137: 191-196.

Garcia-Saez AJ, Chiantia S, Schwille P. 2007. Effect of line tension on the lateral organization of lipid membranes. J Biol Chem 282: 33537-33544.

Gauthier NC, Masters TA, Sheetz MP. 2012. Mechanical feedback between membrane tension and dynamics. Trends Cell Biol 22: 527-535.

* Gautreau A, Oguievetskaia K, Ungermann C. 2014. Function and regulation of the endosomal fusion and fission machineries. Cold Spring Harb Perspect Biol doi: 10.1101/ cshperspect.a016832.

Gonnord P, Blouin CM, Lamaze C. 2012. Membrane trafficking and signaling: Two sides of the same coin. Semin Cell Dev Biol 23: 154-164.

Goswami D, Gowrishankar K, Bilgrami S, Ghosh S, Raghupathy R, Chadda R, Vishwakarma R, Rao M, Mayor S. 2008. Nanoclusters of GPI-anchored proteins are formed by cortical actin-driven activity. Cell 135: 1085-1097.

Gowrishankar K, Ghosh S, Saha SCR, Mayor S, Rao M. 2012. Active remodeling of cortical actin regulates spatiotemporal organization of cell surface molecules. Cell 149: $1353-1367$.

Hansen CG, Nichols BJ. 2009. Molecular mechanisms of clathrin-independent endocytosis. J Cell Sci 122: 1713-1721.

Heimburg T, Jackson AD. 2005. On soliton propagation in biomembranes and nerves. Proc Natl Acad Sci 102: 9790 9795.

Helfrich W. 1973. Elastic properties of lipid bilayers: Theory and possible experiments. Zur Naturforschung 28c: 693703.

Henne WM, Kent HM, Ford MGJ, Hegde BG, Daumke O, Butler PJG, Mittal R, Langen R, Evans PR, McMahon HT 2007. Structure and analysis of FCHo2 F-BAR domain: A dimerizing and membrane recruitment module that affects membrane curvature. Structure 15: 839-852.

Henne WM, Boucrot E, Meinecke M, Evergren E, Vallis Y, Mittal R, McMahon HT. 2010. FCHo proteins are nucleators of clathrin-mediated endocytosis. Science 328: 1281-1284. 
L. Johannes et al.

* Henne WM, Stenmark H, Emr SD. 2013. Molecular mechanisms of the membrane sculpting ESCRT pathway. Cold Spring Harb Perspect Biol 5: a016766.

Howes MT, Mayor S, Parton RG. 2010. Molecules, mechanisms, and cellular roles of clathrin-independent endocytosis. Curr Opin Cell Biol 22: 519-527.

Hu J, Shibata Y, Voss C, Shemesh T, Li Z, Coughlin M, Kozlov MM, Rapoport TA, Prinz WA. 2008. Membrane proteins of the endoplasmic reticulum induce high-curvature tubules. Science 319: 1247-1250.

Idrissi FZ, Blasco A, Espinal A, Geli MI. 2012. Ultrastructural dynamics of proteins involved in endocytic budding. Proc Natl Acad Sci 109: E2587-E2594.

Johannes L, Mayor S. 2010. Induced domain formation in endocytic invagination, lipid sorting, and scission. Cell 142: $507-510$.

Julicher F, Lipowsky R. 1993. Domain induced budding of vesicles. Phys Rev Lett 70: 2964-2967.

Kaksonen M, Toret CP, Drubin DG. 2005. A modular design for the clathrin- and actin-mediated endocytosis machinery. Cell 123: 305-320.

Kaksonen M, Toret CP, Drubin DG. 2006. Harnessing actin dynamics for clathrin-mediated endocytosis. Nat Rev Mol Cell Biol 7: 404-414.

Kirkham M, Fujita A, Chadda R, Nixon SJ, Kurzchalia TV, Sharma DK, Pagano RE, Hancock JF, Mayor S, Parton RG. 2005. Ultrastructural identification of uncoated caveolin-independent early endocytic vehicles. J Cell Biol 168: $465-476$.

Koster G, VanDuijn M, Hofs B, Dogterom M. 2003. Membrane tube formation from giant vesicles by dynamic association of motor proteins. Proc Natl Acad Sci 100: 15583-15588.

Kukulski W, Schorb M, Welsch S, Picco A, Kaksonen M, Briggs JAG. 2011. Correlated fluorescence and 3D electron microscopy with high sensitivity and spatial precision. J Cell Biol 192: 111-119.

Kukulski W, Schorb M, Kaksonen M, Briggs JA. 2012. Plasma membrane reshaping during endocytosis is revealed by time-resolved electron tomography. Cell 150: 508-520.

Kumari S, Mg S, Mayor S. 2010. Endocytosis unplugged: Multiple ways to enter the cell. Cell Res 20: 256-275.

Leibler S. 1986. Curvature instability in membranes. J Phys I 47: 507-516.

Lingwood D, Ries J, Schwille P, Simons K. 2008. Plasma membranes are poised for activation of raft phase coalescence at physiological temperature. Proc Natl Acad Sci 105: $10005-10010$.

Lipowsky R. 1993. Domain-induced budding of fluid membranes. Biophys J 64: 1133-1138.

Liu AP, Fletcher DA. 2006. Actin polymerization serves as a membrane domain switch in model lipid bilayers. Biophys J 91: 4064-4070.

Liu J, Kaksonen M, Drubin DG, Oster G. 2006. Endocytic vesicle scission by lipid phase boundary forces. Proc Natl Acad Sci 103: 10277-10282.

Liu J, Sun Y, Drubin DG, Oster GF. 2009. The mechanochemistry of endocytosis. PLoS Biol 7: e1000204.

Marsh D. 2006. Elastic curvature constants of lipid monolayers and bilayers. Chem Phys Lipids 144: 146-159.
Massol RH, Larsen JE, Kirchhausen T. 2005. Possible role of deep tubular invaginations of the plasma membrane in MHC-I trafficking. Exp Cell Res 306: 142-149.

Masuda M, Mochizuki N. 2010. Structural characteristics of BAR domain superfamily to sculpt the membrane. Sem Cell Dev Biol 21: 391-398.

Mattila PK, Pykalainen A, Saarikangas J, Paavilainen VO, Vihinen H, Jokitalo E, Lappalainen P. 2007. Missing-inmetastasis and IRSp53 deform $\mathrm{PI}(4,5) \mathrm{P}_{2}$-rich membranes by an inverse BAR domain-like mechanism. J Cell Biol 176: 953-964.

McMahon HT, Boucrot E. 2011. Molecular mechanism and physiological functions of clathrin-mediated endocytosis. Nat Rev Mol Cell Biol 12: 517-533.

McMahon HT, Gallop JL. 2005. Membrane curvature and mechanisms of dynamic cell membrane remodelling. Nature 438: 590-596.

McMahon HT, Mills IG. 2004. COP and clathrin-coated vesicle budding: Different pathways, common approaches. Curr Opin Cell Biol 16: 379-391.

Merrifield CJ. 2004. Seeing is believing: Imaging actin dynamics at single sites of endocytosis. Trends Cell Biol 14: 352-358.

* Merrifield C, Kaksonen M. 2014. Endocytic accessory factors and regulation of clathrin-mediated endocytosis. Cold Spring Harb Perspect Biol doi: 10.1101/cshperspect.a016733.

Mettlen M, Pucadyil T, Ramachandran R, Schmid SL. 2009. Dissecting dynamin's role in clathrin-mediated endocytosis. Biochem Soc Trans 37: 1022-1026.

Mim C, Unger VM. 2012. Membrane curvature and its generation by BAR proteins. Trends Biochem Sci 37: 526533.

Morlot S, Galli V, Klein M, Chiaruttini N, Manzi J, Humbert F, Dinis L, Lenz M, Cappello G, Roux A. 2012. Membrane shape at the edge of the dynamin helix sets location and duration of the fission reaction. Cell 151: 619-629.

Pan J, Tristram-Nagle S, Nagle JF. 2009. Effect of cholesterol on structural and mechanical properties of membranes depends on lipid chain saturation. Phys Rev E 80: 021931.

Peter BJ, Kent HM, Mills IG, Vallis Y, Butler PJ, Evans PR, McMahon HT. 2004. BAR domains as sensors of membrane curvature: The amphiphysin BAR structure. Science 303: 495-499.

Polozov IV, Bezrukov L, Gawrisch K, Zimmerberg J. 2008. Progressive ordering with decreasing temperature of the phospholipids of influenza virus. Nat Chem Biol 4: $248-$ 255.

Pucadyil TJ, Schmid SL. 2008. Real-time visualization of dynamin-catalyzed membrane fission and vesicle release. Cell 135: 1263-1275.

Pucadyil TJ, Schmid SL. 2009. Conserved functions of membrane active GTPases in coated vesicle formation. Science 325: 1217-1220.

Rawicz W, Olbrich KC, McIntosh T, Needham D, Evans E. 2000. Effect of chain length and unsaturation on elasticity of lipid bilayers. Biophys J 79: 328-339.

Römer W, Berland L, Chambon V, Gaus K, Windschiegl B, Tenza D, Aly MR, Fraisier V, Florent J-C, Perrais D, et al. 2007. Shiga toxin induces tubular membrane invaginations for its uptake into cells. Nature 450: 670-675. 
Römer W, Pontani LL, Sorre B, Rentero C, Berland L, Chambon V, Lamaze C, Bassereau P, Sykes C, Gaus K, et al. 2010. Actin dynamics drive membrane reorganization and scission in clathrin-independent endocytosis. Cell 140: $540-553$.

Rossman JS, Lamb RA. 2011. Influenza virus assembly and budding. Virology 411: 229-236.

Roux A, Cappello G, Cartaud J, Prost J, Goud B, Bassereau P. 2002. A minimal system allowing tubulation with molecular motors pulling on giant liposomes. Proc Natl Acad Sci 99: 5394-5399.

Roux A, Cuvelier D, Nassoy P, Prost J, Bassereau P, Goud B. 2005. Role of curvature and phase transition in lipid sorting and fission of membrane tubules. EMBO J 24: 1537-1545.

Roux A, Uyhazi K, Frost A, De Camilli P. 2006. GTP-dependent twisting of dynamin implicates constriction and tension in membrane fission. Nature 441: 528-531.

Roux A, Koster G, Lenz M, Sorre B, Manneville J-B, Nassoy P, Bassereau P. 2010. Membrane curvature controls dynamin polymerization. Proc Natl Acad Sci 107: 4141-4146.

Safouane M, Berland L, Callan-Jones A, Sorre B, Römer W, Johannes L, Toombes GE, Bassereau P. 2010. Lipid cosorting mediated by Shiga toxin induced tubulation. Traffic 11: 1519-1529.

Sarasij RC, Mayor S, Rao M. 2007. Chirality induced budding: A raft-mediated mechanism for endocytosis and morphology of caveolae? Biophys J 92: 3140-3158.

Schmid SL, Frolov VA. 2011. Dynamin: Functional design of a membrane fission catalyst. Ann Rev Cell Dev Biol 27: 79-105.

Semrau S, Schmidt T. 2009. Membrane heterogeneityFrom lipid domains to curvature effects. Soft Matter 5: 3174-3186.

Sens P, Johannes L, Bassereau P. 2008. Biophysical view on protein-induced membrane deformations in endocytosis. Curr Opin Cell Biol 20: 476-482.

Shnyrova AV, Bashkirov PV, Akimov SA, Pucadyil TJ, Zimmerberg J, Schmid SL, Frolov VA. 2013. Geometric catalysis of membrane fission driven by flexible dynamin rings. Science 339: 1433-1436.

Singh P, Mahata P, Baumgart T, Das SL. 2012. Curvature sorting of proteins on a cylindrical lipid membrane tether connected to a reservoir. Phys Rev E 85: 051906.

Sinha B, Köster D, Ruez R, Gonnord P, Bastiani M, Abankwa D, Stan RV, Butler-Browne G, Vedie B, Johannes L, et al. 2011. Cells respond to mechanical stress by rapid disassembly of caveolae. Cell 144: 402-413.
Solon J, Gareil O, Bassereau P, Gaudin Y. 2005. Membrane deformations induced by the matrix protein of vesicular stomatitis virus in a minimal system. J Gen Virol 86: 3357-3363.

Sorre B, Callan-Jones A, Manneville JB, Nassoy P, Joanny JF, Prost J, Goud B, Bassereau P. 2009. Curvature-driven lipid sorting needs proximity to a demixing point and is aided by proteins. Proc Natl Acad Sci 106: 5622-5626.

Sorre B, Callan-Jones A, Manzi J, Goud B, Prost J, Bassereau P, Roux A. 2012. Nature of curvature coupling of amphiphysin with membranes depends on its bound density. Proc Natl Acad Sci 109: 173-178.

Stachowiak JC, Hayden CC, Sasaki DY. 2010. Steric confinement of proteins on lipid membranes can drive curvature and tubulation. Proc Natl Acad Sci 107: 7781-7786.

Stachowiak JC, Schmid EM, Ryan CJ, Ann HS, Sasaki DY, Sherman MB, Geissler PL, Fletcher DA, Hayden CC. 2012. Membrane bending by protein-protein crowding. Nat Cell Biol 14: 944-949.

Stagg SM, LaPointe P, Balch WE. 2007. Structural design of cage and coat scaffolds that direct membrane traffic. Curr Opin Struct Biol 17: 221-228.

Stechmann B, Bai SK, Gobbo E, Lopez R, Merer G, Pinchard S, Panigai L, Tenza D, Raposo G, Beaumelle B, et al. 2010 Inhibition of retrograde transport protects mice from lethal ricin challenges. Cell 141: 231-242.

Takei K, Haucke V, Slepnev V, Farsad K, Salazar M, Chen H, De Camilli P. 1998. Generation of coated intermediates of clathrin-mediated endocytosis on protein-free liposomes. Cell 94: 131-141.

Taylor MJ, Perrais D, Merrifield CJ. 2011. A high precision survey of the molecular dynamics of mammalian clathrin mediated endocytosis. PloS Biol 9: e1000604.

Taylor MJ, Lampe M, Merrifield CJ. 2012. A feedback loop between dynamin and actin recruitment during clathrinmediated endocytosis. PloS Biol 10: e1001302.

Tian A, Baumgart T. 2009. Sorting of lipids and proteins in membrane curvature gradients. Biophys J 96: 2676-2688.

Tian A, Johnson C, Wang W, Baumgart T. 2007. Line tension at fluid membrane domain boundaries measured by micropipette aspiration. Phys Rev Lett 98: 208102-208104.

Watkins EB, Miller CE, Majewski J, Kuhl TL. 2011. Membrane texture induced by specific protein binding and receptor clustering: Active roles for lipids in cellular function. Proc Natl Acad Sci 108: 6975-6980.

Welsch S, Müller B, Kräusslich H-G. 2007. More than one door-Budding of enveloped viruses through cellular membranes. FEBS Lett 581: 2089-2097. 


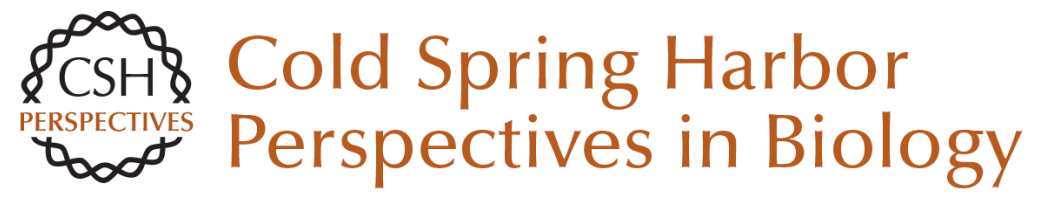

\section{Bending "On the Rocks"--A Cocktail of Biophysical Modules to Build Endocytic Pathways}

Ludger Johannes, Christian Wunder and Patricia Bassereau

Cold Spring Harb Perspect Biol 2014; doi: 10.1101/cshperspect.a016741

Subject Collection Endocytosis

Endocytosis: Past, Present, and Future Sandra L. Schmid, Alexander Sorkin and Marino Zerial

Rab Proteins and the Compartmentalization of the Endosomal System Angela Wandinger-Ness and Marino Zerial

Cargo Sorting in the Endocytic Pathway: A Key Regulator of Cell Polarity and Tissue Dynamics Suzanne Eaton and Fernando Martin-Belmonte

Unconventional Functions for Clathrin, ESCRTs, and Other Endocytic Regulators in the

Cytoskeleton, Cell Cycle, Nucleus, and Beyond:

Links to Human Disease

Frances M. Brodsky, R. Thomas Sosa, Joel A. Ybe, et al.

Endocytosis of Viruses and Bacteria Pascale Cossart and Ari Helenius

Lysosomal Adaptation: How the Lysosome Responds to External Cues Carmine Settembre and Andrea Ballabio

Reciprocal Regulation of Endocytosis and Metabolism Costin N. Antonescu, Timothy E. McGraw and Amira Klip

Endocytosis and Autophagy: Exploitation or Cooperation?

Sharon A. Tooze, Adi Abada and Zvulun Elazar
Imaging and Modeling the Dynamics of

Clathrin-Mediated Endocytosis

Marcel Mettlen and Gaudenz Danuser

Endocytic Accessory Factors and Regulation of

Clathrin-Mediated Endocytosis

Christien J. Merrifield and Marko Kaksonen

The Complex Ultrastructure of the Endolysosomal

System Judith Klumperman and Graça Raposo

The Biogenesis of Lysosomes and

Lysosome-Related Organelles

J. Paul Luzio, Yvonne Hackmann, Nele M.G.

Dieckmann, et al.

Endocytosis, Signaling, and Beyond Pier Paolo Di Fiore and Mark von Zastrow

Clathrin-Independent Pathways of Endocytosis Satyajit Mayor, Robert G. Parton and Julie G. Donaldson

The Role of Endocytosis during Morphogenetic Signaling Marcos Gonzalez-Gaitan and Frank Jülicher

Role of Endosomes and Lysosomes in Human Disease Frederick R. Maxfield

For additional articles in this collection, see http://cshperspectives.cshlp.org/cgi/collection/

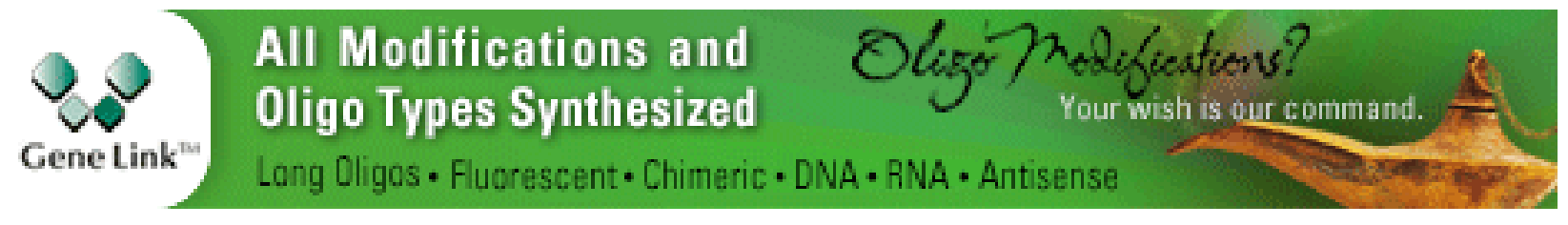


For additional articles in this collection, see http://cshperspectives.cshlp.org/cgi/collection/

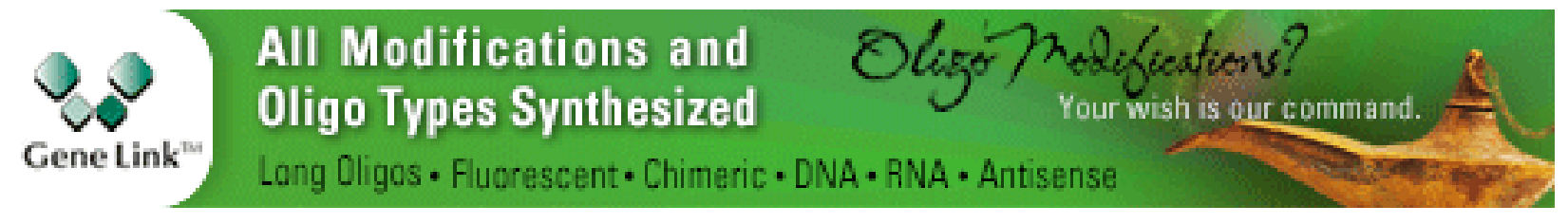

Copyright @ 2014 Cold Spring Harbor Laboratory Press; all rights reserved 